\title{
Embarazo Prolongado. Diagnóstico y Evaluación
}

\author{
Universidad Nacional de Colombia \\ Facultad de Medicina
}

Departamento de Ginecología y Obstetricia
Instituto Materno-Infantil Bogotá

Dres.: Juan Alvaro Salazar G: ${ }^{1}$ Carlos Julio Pacheco C. ${ }^{2}$ Héctor Ulloque 3

Armando Guzmán N.4

Asesoría y Supervisión Profesor Alvaro Velasco Ch.

\section{APLICACION DE LAS LECTURAS DE DENSIDAD OPTICA EN LIOUIDO AMNIOTICO A $650 \mathrm{~nm}$}

El manejo del embarazo prolongado (EP) cuenta en la actualidad con diferentes medios diagnósticos que garantizan la detección oportuna del cuadro de Insuficiencia Placentaria (IP) a que puede llevar.

Se ha demostrado una mayor incidencia de morbimortalidad perinatal en el postérmino como consecuencia de una IP progresiva (12) que plantea dos posibilidades, la monitorización periódica cuidadosa, de la paciente en quien se sospecha esta situación clínica y el diag-

1. Residente III Ginecoobstetricia

2. Residente III Ginecoobstetricia

3. Profesor Asistente Pediatría

4. Jefe de la Sección de Química H.S.J.D. nóstico confiable del sobrepaso del término. La primera es la más aceptada $(5,7)$, e incluye mediciones hormonales y pruebas con la frecuencia cardíaca fetal, que ponen en evidencia la condición fetal actual; esta conducta exige recursos de tipo humano y tecnológico especializados y debe incluir la gran mayoría de mujeres que han consultado por sobrepasar su fecha probable de parto, aunque en muchas de ellas se concluya después un error en el cálculo de tal fecha o la iniciación del embarazo después de un período preovulatorio prolongado. La segunda requiere disponer de una medida diagnóstica de la edad gestacional (EG), aplicable en el postérmino; nuestro trabajo propone la densidad óptica (DO) del líquido amniótico (LA) para tal fin. 
La amniocentesis había sido propuesta como medida diagnóstica y de evaluación del EP tomando como base la asociación entre mayores índices de morbimortalidad perinatal y la obtención de LA contaminado por meconio (1), y de otra parte el mayor porcentaje de líquidos con meconio en el EP. Análisis más objetivos sobre el "Iíquido meconiado" han reconocido una mayor incidencia en el EP, pero descartan su utilidad como medida de diagnóstico o pronóstico $(4,7)$. La cuantificación de diversos componentes del LA han permitido evaluar la $E G$, la madurez fetal y pulmonar, sin que se logre elegir algún parámetro que mantenga correlación definida con la EG después de las 40 semanas; sin embargo, las lecturas de DO a $650 \mathrm{~nm}$ en el último trimestre de embarazo demostraron excelente correlación con la EG y tendencia al ascenso aún en el postérmino, en trabajo realizado por Salazar, Pacheco y Colab. (9). Este parámetro que hasta ahora demuestra gran utilidad en la predicción de la madurez pulmonar fetal a nivel experimental y clínico $(6,10,11)$, ofrece además la posibilidad de hacer un diagnóstico bien aproximado de la EG después de la semana 34 , aplicando la fórmula de la curva de regresión obtenida con los valores de DO (9).

$$
E G=45-\frac{1}{0,07-0,7\left(D_{0650 n m)}\right.}
$$

Este trabajo (9) fue elaborado con 84 muestras entre las 27 y 43 semanas de gestación, entre las cuales solo se incluían 7 con 294 días o más; por consiguiente consideramos de utilidad recolectar nuevos casos de EP que nos permitieran observar que pasa con la DO después de la semana 42 y su posibilidad diagnóstica, si comprobamos una diferencia significativa entre las cifras del embarazo a término y el prolongado.

Un objetivo secundario será evaluar el riesgo que constituye la prolongación del embarazo, sometiendo los casos seleccionados a pruebas de función placentaria mediante la monitoría de la frecuencia cardíaca fetal, para comparar las tasas de IP diagnosticada con la publicada por otros autores.

\section{MATERIAL Y METODOS}

Durante un período aproximado de un año seleccionamos pacientes de la Consulta de Admisión del Instituto Materno Infantil de Bogotá; anotamos su motivo de consulta, sus antecedentes de importancia y tomamos como requisitos básicos una fecha de última regla confiable, ciclos menstruales regulares, ausencia de patología médica u obstétrica importantes sobreagregadas, $y$ por último, amenorrea mayor de 300 días contabilizados a partir del primer día de su última mestruación. No se incluyeron otros requisitos para corroborar la EG calculada en el momento de la amniocentesis, como la progresión de la altura uterina, la fecha en que la paciente refiere movimientos fetales o en la cual se escuchan por primera vez los ruidos cardíacos fetales, ya que se hubiera requerido un control prenatal muy detallado; ello podría ser motivo de otra investigación que busque perfeccionar los resultados del presente trabajo. Un requisito aplicado de manera retrospectiva a los casos estudiados fue la EG calculada en el recién nacido por un pediatra por el método de Capurro (2) no inferior a 40 semanas.

Practicamos amniocentesis transabdominal, en algunos casos previa ecografía para localización placentaria, medida del diámetro biparietal (DBP) y detección de anomalías importantes. Obtenido el LA anotamos sus características macroscópicas, descartamos las muestras contaminadas por sangre y procedimos a la centrifugación de la muestra a $\mathbf{3 0 0 0}$ rpm durante 10 minutos para luego realizar las lecturas de DO en un espectrofotómetro "Coleman" a $650 \mathrm{~nm}$. En 
algunos casos realizamos la prueba de la espuma estable para corroborar la madurez fetal pulmonar.

La segunda etapa fue la evaluación de la función placentaria mediante las técnicas descritas para la prueba sin estado de alarma o de no estres (6) y la prueba de oxitocina $(3,8)$, anotando la línea base, la variabilidad y las fluctuaciones de la frecuencia cardíaca fetal de tipo periódico y no periódico.

En base a los resultados de la propia DO, la prueba de la espuma estable y la historia clínica, decidimos desembarazar cada paciente; la vía del parto y el tipo de monitorización que se proporcionó dependía de los antecedentes obstétricos, los resultados de las pruebas de evaluación de la función placentaria y la evolución del trabajo de parto $\mathrm{y}$ del cuello uterino. Tuvimos en cuenta las condiciones del parto y especialmente el Apgar y el peso del recién nacido.

Las cifras de DO fueron analizadas junto con las del trabajo ya publicado (9) tomando los valores superiores a las 38 semanas para comparar los datos logrados entre el término (38 a 42 semanas) y el postérmino.

\section{RESULTADOS}

Fueron estudiadas 16 pacientes, cuyas características se pueden observar en los cuadros 1 a 4 . El motivo de consulta anotado en 14 casos tuvo predominio por la prolongación del embarazo; las edades registradas oscilaron entre $17 \mathrm{y}$ 41 años y promedió en 23,5. Existió solo una discreta mayoría de pacientes multíparas. La altura uterina varió entre 30 y $36 \mathrm{~cm}$, con promedio de $32,6 \mathrm{~cm}$. El DBP medido en 8 estudios ecográficos estuvo entre 9,4 y $10 \mathrm{~cm}$ mediando en 9,75 ; solo en un caso $(12,5 \%)$ se observaron alteraciones de la homogeneidad placentaria compatibles con EP.
CARACTERISTICAS

DE LAS PACIENTES SELECCIONADAS

Cuadro No. 1

\begin{tabular}{|lc|}
\hline \multicolumn{2}{|c|}{ MOTIVo DE CONSULTA } \\
\hline Prolongación del embarazo & 8 \\
Preparto & 5 \\
Otros & 1 \\
Total & 14 \\
\hline
\end{tabular}

Cuadro No. 2

\begin{tabular}{|lc|}
\hline \multicolumn{2}{|c|}{ EDAD (años) } \\
\hline 20 o menos & 4 \\
20 a 25 & 5 \\
25 a 30 & 5 \\
30 o más & 1 \\
Total & 15 \\
$\bar{X}: 23.5$ & \\
\hline
\end{tabular}

Cuadro No. 3

\begin{tabular}{|lr|}
\hline \multicolumn{2}{|c|}{ PARIDAD } \\
\hline Primigestante & 5 \\
Secundigestante & 1 \\
Multigestante & 8 \\
Total & 14 \\
\hline
\end{tabular}

Cuadro No. 4

\begin{tabular}{|c|c|}
\hline \multicolumn{2}{|c|}{ ALTURA UTERINA (cm) } \\
\hline menor de 30 & 7 \\
\hline 30 a 32 & 8 \\
\hline mayor de 32 & 5 \\
\hline Total & 14 \\
\hline- & \\
\hline$x: 32,6$ & \\
\hline
\end{tabular}


La amniocentesis se realizó en 16 pacientes, obteniendo líquido de aspecto grumoso blanquecino, excepto en 3 casos $(18.75 \%)$ en que se apreció tinte meconial franco. Las lecturas de DO se practicaron en 15 líquidos y sus resultados se anotan junto con el tiempo de amenorrea correspondiente en el cuadro No. 5.

\section{Cuadro No. 5}

\section{LECTURAS DE DENSIDAD OPTICA EN} LIQUIDO AMNIOTICO a $650 \mathrm{~nm}$

\begin{tabular}{|c|l|}
\hline Tiempo de amenorrea & \multicolumn{1}{|c|}{ DO } \\
\hline 300 & 0,553 \\
301 & 0,357 \\
302 & 0,585 \\
305 & 0,367 \\
305 & 0,638 \\
305 & 0,824 \\
305 & 0,444 \\
308 & 0,207 \\
309 & 0,509 \\
310 & 0,45 \\
311 & 0,328 \\
311 & 0,42 \\
314 & 0,367 \\
315 & 0,377 \\
331 & 0,310 \\
\hline
\end{tabular}

En el cuadro No. 6 y la gráfica No. 1 aparecen los datos del presente trabajo junto con los tomados de la investigación ya publicada (9), correspondiendo a esta última todos los valores inferiores a 300 días de amenorrea y 4 de los que están por encima de este tiempo de embarazo. Sobre la gráfica se trazan dos líneas una que divide los datos de EG en los tiempos inferiores a 290 días y los que están por encima $y$ otra que separa las cifras de DO en inferiores a 0,3 y superiores.
Gráfica No. 7

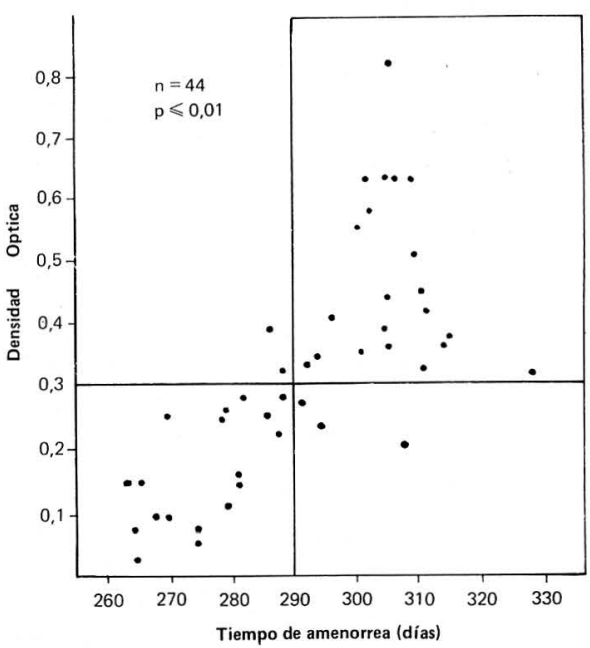

VALORES DE DENSIDAD OPTICA A $650 \mathrm{~nm}$ EN LIQUIDO AMNIOTICO EN EL EMBARAZO A TERMINO Y PROLONGADO

La distribución resultante se observa en el cuadro No. 7: existen 21 casos con tiempo de amenorrea inferior a 290 días de los cuales $18(90.0 \%)$ tienen valores de DO inferior a 0,3 , y se cuentan 24 casos con más de 290 días entre los que hay $21(87.5 \%)$ mayores de 0,3 en su DO.

Cuadro No. 7

\section{DISTRIBUCION DE LA EDAD}

GESTACIONAL DE ACUERDO A

\section{LAS CIFRAS DE DENSIDAD OPTICA}

\begin{tabular}{|l|c|r|c|}
\hline \multirow{2}{*}{$\begin{array}{c}\text { Tiempo de } \\
\text { amenorrea } \\
\text { (dias) }\end{array}$} & \multicolumn{2}{|c|}{ Densidad optica a $650 \mathrm{~nm}$} & \multirow{2}{*}{ Total } \\
\cline { 2 - 3 } & Menor de 0,3 & Mayor de 0,3 & \\
\hline $\begin{array}{l}\text { Menor de } \\
290\end{array}$ & $\begin{array}{c}18 \\
(90 \%)\end{array}$ & $\begin{array}{c}2 \\
(10 \%)\end{array}$ & 20 \\
\hline $\begin{array}{l}\text { Mayor de } \\
290\end{array}$ & $\begin{array}{c}3 \\
(12,5 \%)\end{array}$ & $\begin{array}{c}21 \\
(87,5 \%)\end{array}$ & 24 \\
\hline
\end{tabular}


Cuadro No. 6

COMPARACION DE LAS CIFRAS DE DENSIDAD OPTICA EN LIQUIDO

AMNIOTICO A 650 nm EN EMBARAZOS A TERMINO Y PROLONGADOS

\begin{tabular}{|c|c|}
\hline Tiempo de amenorrea & DO \\
\hline $263^{\prime}$ & 0,143 \\
\hline $264^{\prime}$ & 0,071 \\
\hline $264^{\prime}$ & 0,022 \\
\hline $265^{\prime}$ & 0,143 \\
\hline $267^{\prime}$ & 0,092 \\
\hline $269^{\prime}$ & 0,092 \\
\hline $271^{\prime}$ & 0,262 \\
\hline $274^{\prime}$ & 0,071 \\
\hline $274^{\prime}$ & 0,048 \\
\hline $278^{\prime}$ & 0,252 \\
\hline $279^{\prime}$ & 0,252 \\
\hline $279^{\prime}$ & 0,108 \\
\hline $281^{\prime}$ & 0,155 \\
\hline $281^{\prime}$ & 0,149 \\
\hline $282^{\prime}$ & 0,284 \\
\hline $285^{\prime}$ & 0,252 \\
\hline $286^{\prime}$ & 0,398 \\
\hline $288^{\prime}$ & 0,229 \\
\hline $288^{\prime}$ & 0,328 \\
\hline $289^{\prime}$ & 0,284 \\
\hline $291^{\prime}$ & 0,277 \\
\hline $292^{\prime}$ & 0,338 \\
\hline
\end{tabular}

\begin{tabular}{|c|c|}
\hline Tiempo de amenorrea & DO \\
\hline $294^{\prime}$ & 0,347 \\
\hline $295^{\prime}$ & 0,244 \\
\hline $296^{\prime}$ & 0,409 \\
\hline 300 & 0,553 \\
\hline 301 & 0,357 \\
\hline $302^{\prime}$ & 0,638 \\
\hline 302 & 0,585 \\
\hline $305^{\prime}$ & 0,387 \\
\hline 305 & 0,367 \\
\hline 305 & 0,824 \\
\hline 305 & 0,444 \\
\hline 305 & 0,632 \\
\hline $306^{\prime}$ & 0,638 \\
\hline 308 & 0,207 \\
\hline $309^{\prime}$ & 0,638 \\
\hline 309 & 0,509 \\
\hline 310 & 0,45 \\
\hline 311 & 0,42 \\
\hline 311 & 0,328 \\
\hline 314 & 0,367 \\
\hline 315 & 0,377 \\
\hline 331 & 0,310 \\
\hline
\end{tabular}

(') Datos tomados de la investigación anterior (9)

El estudio de la función placentaria se realizó mediante la prueba sin estado de alarma en 13 casos y en 12 de ellos con la prueba de oxitocina, los resultados se tabularon en los cuadros 8 y 9 . La primera registró 5 pruebas no reactivas $(38,5 \%)$ y la segunda 3 positivas $(25 \%)$. Los datos de los recién nacidos se anotan en los cuadros 10,11 y 12 . El peso al nacer osciló entre 2380 y 3650 , promedio de $3143 \mathrm{~g}$ en 13 casos. El Apgar se midió en 14 casos y de ellos 2 fueron de 8 y 2 de 6 . La evaluación por el método de Capurro se practicó en 11 nacidos y varió entre 40 y 42 semanas. Nueve de ellos habían sido sometidos a pruebas de función placentaria. En los cuadros 13 y 14 se distribuyen los resultados de la 
PRUEBAS DE FUNCION PLACENTARIA

Cuadro No. 8

\section{PRUEBA SIN ESTADO DE ALARMA}

\begin{tabular}{|lrr|}
\hline Reactivas & 8 & $(61,54 \%)$ \\
No Reactivas & 5 & $(38.46 \%)$ \\
Total & 13 & \\
\hline
\end{tabular}

Cuadro No. 9

PRUEBA DE OXITOCINA

\begin{tabular}{|lrl|}
\hline Positivas & 3 & $(25 \%)$ \\
Negativas & 8 & $(74,17 \%)$ \\
Dudosas & 1 & $(0.83 \%)$ \\
Total & 12 & \\
\hline
\end{tabular}

evaluación placentaria de acuerdo a la edad gestacional calculada por el pediatra, en dos grupos: los de EG menor de 41,5 y los que superaron este cálculo. En solo una de las evaluaciones se anotaron signos incipientes de postmadurez, un caso al que se diagnosticó IP y Apgar de 6.

\section{CARACTERISTICAS \\ DE LOS RECIEN NACIDOS EN LOS CASOS ESTUDIADOS}

Cuadro No. 10

\begin{tabular}{|lr|}
\hline \multicolumn{2}{|c|}{ Peso (gr) } \\
\hline Menor de 3000 & 3 \\
3000 a 3500 & 9 \\
mayor de 3500 & 1 \\
Total & 13 \\
\hline
\end{tabular}

Cuadro No. 11

\begin{tabular}{|cc|}
\hline \multicolumn{2}{|c|}{ Apgar } \\
\hline 10 & 8 \\
8 & 2 \\
6 & 2 \\
Total & 12 \\
\hline
\end{tabular}

Cuadro No. 12

\section{RELACION ENTRE TIEMPO DE AMENORREA Y LA EVALUACION DEL RECIEN NACIDO POR EL METODO DE CAPURRO}

\begin{tabular}{|c|c|c|c|c|c|c|}
\hline $\begin{array}{l}\text { Tiempo de } \\
\text { amenorrea } \\
\text { (días) }\end{array}$ & $\begin{array}{l}\text { Formación } \\
\text { del } \\
\text { Pezón }\end{array}$ & $\begin{array}{l}\text { Nódulo } \\
\text { Mamario }\end{array}$ & $\begin{array}{l}\text { Forma } \\
\text { de la } \\
\text { oreja }\end{array}$ & $\begin{array}{l}\text { Textura } \\
\text { de la } \\
\text { piel }\end{array}$ & $\begin{array}{l}\text { Pliegues } \\
\text { plant. }\end{array}$ & $\begin{array}{l}\text { Edad } \\
\text { Gestac. }\end{array}$ \\
\hline 300 & 15 & 10 & 24 & 15 & 20 & 41 \\
\hline 301 & 15 & 15 & 24 & 15 & 20 & 41,5 \\
\hline 302 & 15 & 15 & 24 & 15 & 15 & 40,5 \\
\hline 305 & 15 & 10 & 24 & 15 & 20 & 41 \\
\hline 308 & 15 & 12 & 24 & 18 & 20 & 41,8 \\
\hline 311 & 15 & 12 & 24 & 18 & 20 & 42 \\
\hline 311 & 15 & 10 & 24 & 15 & 15 & 40 \\
\hline 315 & 15 & 15 & 24 & 17 & 20 & 42 \\
\hline 331 & 15 & 10 & 24 & 15 & 15 & 40,5 \\
\hline 332 & 15 & 15 & 20 & 15 & 15 & 40,5 \\
\hline
\end{tabular}


Cuadro No. 13

RELACION ENTRE LAS PRUEBAS DE FUNCION PLACENTARIA Y LA EDAD GESTACIONAL CALCULADA AL RECIEN NACIDO POR EL METODO DE CAPURRO

\begin{tabular}{|l|c|c|c|c|}
\hline \multirow{2}{*}{$\begin{array}{l}\text { Edad } \\
\text { Gestacional } \\
\text { (semanas) }\end{array}$} & \multicolumn{2}{|c|}{ Prueba de no esfuerzo } & \multicolumn{2}{c|}{ Prueba de Oxitocina } \\
\cline { 2 - 5 } & Reactiva & No Reactiva & Negativa & Positiva \\
\hline $\begin{array}{l}\text { menor de } \\
41,5\end{array}$ & 4 & 1 & 5 & 0 \\
\hline $\begin{array}{l}\text { mayor de } \\
41,5\end{array}$ & 1 & 3 & 2 & 2 \\
\hline
\end{tabular}

\section{COMENTARIOS}

El estudio del EP posee muchos aspectos poco clarificados, dificultando la unificación de los criterios terapéuticos para esta entidad. El proceso de envejecimiento placentario y los mecanismos implicados en la iniciación del trabajo de parto influyen de manera especial en la función placentaria, en su capacidad para tolerar un trabajo de parto, en la sensibilidad a los oxitócicos, en la progresión del trabajo de parto y por último en la incidencia del síndrome de postmadurez y la morbimortalidad perinatal. La participación de factores hormonales, inmunológicos, vasculares y mecánicos hacen necesario concluir que lo más conveniente es la evaluación individual de cada caso de EP. El problema inmediato lo constituye la selección de las pacientes que deben vigilarse, pues bien sabemos que muchas de las pacientes que refieren períodos de amenorrea gestacional excesivos, no poseen un EP y que solo en pocos casos el exámen clínico es capaz de descartar esta situación.

Demostrada la tendencia definida al aumento de las cifras de DO del LA entre las 34 a 42 semanas de embarazo (9) y en base a los resultados del presente trabajo podemos concluir que el análisis espectrofotométrico constituye un medio de selección valioso de las pacientes que ameritan una evaluación por un diagnóstico de EP. Las cifras de DO obtenidas en la muestra poseen una diferencia estadísticamente significativa con los datos obtenidos a término.

Dado que los dos grupos fueron estudiados en iguales circunstancias, los casos a término tomados del trabajo ya publicado, nos sirven de "blanco" a la presente investigación. La diferencia más significativa $\mathrm{CHi}_{2}=26.26$, se aprecia cuando trazamos la división sobre la EG en los 290 días y sobre la DO en los 0,3. Esta cifra divide la población en dos grupos diferentes, para concluir que los valores menores de 0,3 corresponden a embarazos con menos de 290 días con un $10 \%$ de falsos positivos (para EP), y que los valores mayores indican tiempos de gestación superiores con un $12,5 \%$ de falsos negativos. Muy probablemente esta capacidad predictiva de la DO se demuestre más precisa en futuras investigaciones en que se incluyan todos los criterios de selección posibles a la muestra a estudiar. Los datos de edad, paridad, altura uterina obtenidos en el grupo estudiado no permiten hacer conclusiones definitivas, pero no difieren con los correspondientes a la población a término de nuestro medio. Quizás en muestras más representativas se pueda confirmar las diferencias descritas por otros autores para estas variables en el EP, aunque nunca han sido suficientes, para presumir un diagnóstico.

Nuestro segundo objetivo de evaluar la función placentaria demostró porcentajes importantes de alteración en la reserva respiratoria placentaria $(38,5 \%$ para la prueba sin estado de alarma y $25 \%$ para la prueba de oxitocina). Este resultado esta de acuerdo con las cifras citadas en la revisión de Vorher (12) de 11 a $32 \%$. Obviamente en estos porcentajes además del tamaño de la muestra influye el medio de diagnóstico 
utilizado, de todas maneras está suficientemente demostrado el riesgo que constituye el embarazo verdaderamente prolongado.

Vale la pena subrayar que en nuestra casuística a pesar de no reportarse ningún caso franco de postmadurez se repitieron las importantes tasas de IP reportadas por otros autores. Este síndrome ha sido encontrado en índices de $20 \%$ en el EP.

Nos llamó la atención los cálculos de la EG por el método de Capurro cuyos resultados nunca superaron las 42 semanas a pesar de que nuestra muestra solo incluía períodos de amenorrea superiores a los 300 días, ello nos hace suponer que este método no precisa la prolongación del embarazo sino en la medida en que se insinúen características de postmadurez; sin embargo, nos fue de utilidad para obscrvar como los casos en que se había detectado IP correspondieron casi siempre a recién nacidos a los que se calculó EG mayor de 41,5 semanas, $\mathrm{CHi}^{2}$ = no significativa como se observa en el cuadro 13. Los datos de peso y Apgar recogidos en los recién nacidos por su escaso número carecen de importancia, excepto por la coincidencia de que uno de los puntajes de Apgar más bajos era un caso al que se había diagnosticado IP y resultó ser el único recién nacido a quien se encontraron signos incipientes de postmadurez.

La fórmula propuesta en la investigación anterior (9) para deducir la EG conocidas la cifras de DO a $650 \mathrm{~nm}$ en LA no la intentamos aplicar ante la evidente dispersión de valores que obtuvimos en los casos estudiados en el presente trabajo; no descartamos la posibilidad de obtener una tendencia más definida en los valores de DO al realizar una investigación con pacientes de la propia consulta prenatal y una EG calculada de manera más precisa; sin embargo, no se puede tener mucho optimismo sobre resultados que serán siempre afectados por el volúmen de líquido amniótico y aunque se sabe la progresión al oligoamnios del EP (12) también se acepta que existen múltiples factores que afectan dicho volúmen e indirectamente la DO en embarazos anormales como el EP.

\section{CONCLUSIONES}

La DO del LA a $650 \mathrm{~nm}$ constituye una excelente medida para seleccionar pacientes con un diagnóstico bien aproximado de EP. Mientras se realiza un estudio con una muestra más representativa y seleccionada de una consulta prenatal en que se detallen suficientemente los datos para corroborar la EG calculada, proponemos un valor de DO, 0,3 para concluir que un embarazo es de postérmino, si se supera esta cifra en las lecturas espectrofotométricas a $650 \mathrm{~nm}$. Por nuestro trabajo anterior (9) sabemos que cifras menores además de descartar el EP nos orientan con mucha aproximación sobre la correcta edad del embarazo que tratamos.

Coincidimos en los resultados de otros autores en considerar la paciente con EP como un caso de Alto Riesgo, independientemente de su asociación con un síndrome de postmadurez.

Hecho el diagnóstico de EP somos de la opinión de proceder a desembarazar la paciente de la forma que lo indique su evaluación global, ya que encontramos incremento de los índices de IP con respecto al término. No nos resulta lógico evaluar periódicamente un em: barazo hasta detectar un sufrimiento fetal.

El método de Capurro para evaluar el recién nacido no precisa la prolongación del embarazo sino al aparecer signos de postmadurez; sin embargo, la edad gestacional calculada parece correlacionarse con las cifras de IP diagnosticadas 
durante el embarazo, pudiéndose utilizar como pronóstico del recién nacido. EI $\mathrm{CHi}^{2}$ no es significativo.

\section{RESUMEN}

Se estudian 16 pacientes con amenorrea gestacional de 300 días o mayor, se someten a amniocentesis y su líquido amniótico se centrifuga y lee en el espectrofotómetro a $650 \mathrm{~nm}$; estas lecturas se analizan tomando como "blan$\mathrm{co}^{\prime \prime}$ los resultados de un trabajo publicado por los mismos autores en pacientes a término, deduciendo una diferencia significativa entre las cifras de Densidad Optica a término y postérmino. Se propone una cifra de 0,3 como valor crítico por encima del cual se puede presumir el diagnóstico de embarazo prolongado $y$ por debajo del cual se descarta con muy aceptable confiabilidad, concluyendo la bondad del método para la selección de pacientes a estudio por embarazo prolongado.

Se realizan pruebas evaluatorias de la función placentaria mediante pruebas con la frecuencia cardíaca fetal, que producen cifras de Insuficiencia Placentaria, que coinciden con las publicadas por otros autores y sustentan la necesidad de considerar la entidad como un verdadero caso de Alto Riesgo, aún no coexista con un síndrome de postmadurez. Se anota una posible insensibilidad en el método de Capurro para detectar la prolongación del embarazo cuando no se asocia con el cuadro de postmadurez.

\section{PROLONGATED PREGNANCY: DIAGNOSIS AND TREATMENT}

\section{SUMMARY:}

Sixteen patients with amenorrhea of 300 days or more were studied through amnioscentesis; the liquid was centrifugated and read in the espectrophotometer at $650 \mathrm{~mm}$; the readings were analyzed in the light of previous research conducted by the same authors with patients at term. There is a significant difference in the figures of Optical Density in patients at term and postterm. The authors propose 0,3 as critical value above which diagnosis of prolongated pregnancy is presumed and below which prolongated pregnancy can be

\section{BIBLIOGRAFIA}

1. $\mathrm{COZ}$ L.W. Fetal Dsitress. Aust. N. Az. Obst. Gynecol. 1:99. 1961.

2. CAPURRO H., KONIZHEZKY S., FONSECA D., CALDEYRO-BARCIA R. A dismissed. It appears to be a good method for secreening patients for furthertreatment.

The function of the placenta was studied through evaluation of fetal cardiac frequency, producing figures of insufficiency of the placenta which are confirmed by other publications. It is therefore important to consider this as High Risk, even if no post-maturity syndrome is present. The authors suggest a possible insensitivity of Capurro method to detect prolongated pregnancies when not associated with post-maturity symptoms.

simplified method for diagnosis of gestational age in the newborn infant. J. Ped. 93: 120. 1978.

3. FREEMAN R.K., The use of the oxyto. cin challenge test for antepartum clinical evaluation of uteroplacental respiratory 
reserve. Clinical Opinion. Am. J. Obst. Gynec. 45: 142. 1975.

4. GREEN J.N., PAUL R.H., The Value of Amniocentesis in prolonged pregnancy. Obst. Gynecol. Vol. 51. 3. 1978.

5. KHOUZAMI V.A., JOHNSON J.W., HERNANDEZ E., ROTMENSCH J., FRYE R., DAIKOKU N. Urinary estrogens in postterm pregnancy. Am. J. Obst. Gynec. V.141. 2. Sept. 81.

6. KHOUZAMI V.A., BECK J., JOHNSON J., MITZNER W., LONDON W. Amniotic fluid al sorbance at $650 \mathrm{~nm}$ : A comparison with fetal lung maturity charactes ristics. Am J. Obst. Gynec. 141. 2. Sept. 81.

7. KNOX G.E., HUDDLESTON J.F., FLOWERS C.E., EUBANKS A., and SU. TLIFF G. Management of prolonged pregnancy: Results of a prospective randomized trial. Am. J. Obst. Gynec. 134: 376, 1979.

8. RAY., FREEMAN E.K., PINES S., HESSELGESER R. Clinical experience with the oxitocyn challenge test. Am. J. Obst. Gynec. 114:1. 1972.

9. SALAZAR G.A., PACHECO C. C., VELASCO CH. A., GUZMAN N.A. Deter. minación de los valores normales de densidad óptica en líquido amniótico a $650 \mathrm{~nm}$ en el último trimestre del embarazo. Rev. Col. Obst. Gynec. 32. 3. 1981.

10. SBARRA A.J. et al. Relation between, optical density at $650 \mathrm{~nm}$ and L/S ratio. Obst. Gynecol. Vol. 50: 723.724. 1977.

11. SPELLACY W., BUHI W.C., CRUZ A.C., Assessment of fetal lung madurity; a comparision of Lecitin/Sphingomyelin ratio and teh test of optical density at 400 and $650 \mathrm{~nm}$ Am J. Obst. Gynecol. 134: 528-531. 1979.

12. VORHERR H. Placental insufficiency in relation to postterm pregnancy and fetal postmaturity. A. J. Obst. Gynecol. 123. 67. 1975. 